\title{
Earthquakes, influenza and cycles of Indian kala-azar
}

Christopher Dye*and Daniel M. Wolpert Department of Medical Parasitology, London School of Hygiene and Tropical Medicine, Keppel Street, London, WC1E 7HT, UK

\begin{abstract}
It is suggested that previous data indicate 3 major epidemics of kala-azar in Assam between 1875 and 1950 , with inter-epidemic periods of $30-45$ and 20 years. This deviates from the popular view of regular cycles with a 10-20 year period. A deterministic mathematical model of kala-azar is used to find the simplest explanation for the timing of the 3 epidemics, paying particular attention to the role of extrinsic (drugs, natural disasters, other infectious diseases) versus intrinsic (host and vector dynamics, birth and death rates, immunity) processes in provoking the second. We conclude that, whilst widespread influenza in 1918-1919 may have magnified the second epidemic, intrinsic population processes provide the simplest explanation for its timing and synchrony throughout Assam. The model also shows that the second inter-epidemic period is expected to be shorter than the first, even in the absence of extrinsic agents, and highlights the importance of a small fraction of patients becoming chronically infectious (with post kala-azar dermal leishmaniasis) after treatment during an epidemic.
\end{abstract}

\section{Introduction}

A century of speculation about pattern and process has placed cycles of kala-azar-visceral human leishmaniasis due to Leishmania donovani-among the legends of tropical epidemiology (ROGERS, 1908; MCCOMBIE YOUNG, 1924; NAPIER, 1943; SHORTT, 1945; Sen Gupta, 1951; Peters \& Prasad, 1983; MANSON-BAHR \& APTED, 1982; SANYAL, 1985; ASHFORD \& BETTINI, 1987). All of these authorities, from NAPIER (1943) onwards, have agreed upon a 10-20 year periodicity in Assam Province between 1875 and 1950. The most recent review (ASHFORD \& BETTINI, 1987) recognizes 5 epidemic peaks at 1885 , $1897,1913,1925$ and 1944 in the Goalpara District, and consequently a mean inter-epidemic period of about 15 years. Apparently, insecticides have had a substantial impact on kala-azar in Assam since 1949, but few data are available (SEN GUPTA, 1951; SANYAL et al., 1979).

The search for regular intervals between epidemics - the product of intrinsic population processes-implicitly contradicts the view, expressed most confidently by MCCOMBIE YouNG (1924), that outbreaks were precipitated by extrinsic agentsparticularly the influenza pandemic of 1918-1919. The conflict of opinion is acknowledged by most writers on the subject, but has not been satisfactorily resolved.

In this paper we contribute to the debate by studying the behaviour of a mathematical model

"Correspondence to Dr C. Dye at the above address. whose components distil the essentials of kala-azar epidemiology. We first present the data on kala-azar in Assam given by ROGERS (1908), MCCOMBIE YOUNG (1924) and SEN GUPTA (1951), identifying major epidemics and the associated inter-epidemic periods. Then, investigating non-equilibrium dynamics over a 75 year period, we look for the most parsimonious explanation of the observed pattern of epidemics. Our main conclusion emerges from a comparison of kala-azar dynamics at two spatial levels, each of the Districts and then the whole Province. At the heart of the analysis is the question of whether epidemics of kala-azar were driven primarily by intrinsic or extrinsic processes.

\section{The Data}

The first recorded Assam epidemic began in the early 1880's in the Garo Hills, and proceeded eastwards along the Brahmaputra valley (Fig. 1). By 1900 it had travelled 200-300 miles through the Districts of Goalpara, Kamrup, Nowgong and Darrang (ROGERS, 1908; MCCOMBIE Young, 1924; SEN GuPTA, 1951; Fig. 2). Its impact in these four Districts was devastating and generated, among the other disasters of the period, a marked rise in the fever death rate. Clinical diagnosis was unreliable in these early years (ROGERS, 1908; NAPIER, 1926), but it is said that one-third of the population of Nowgong lost their lives in the epidemic of 1892-1898 (SEN GUPTA, 1951). There then followed, in each District, a 'quiescent phase' (MCCOMBIE YOUNG, 1924); cases were widespread but the incidence was generally low. ASHFORD $\&$ BETTINI (1987) associated the 1897 peak in fever death rate in Goalpara with kala-azar, but RoGERS (1908) attributed it to famine and earthquake, without specifically mentioning kala-azar. The 1913 outbreak in Goalpara was apparently a local, minor precursor to a second epidemic which struck with greatest force in the early 1920 s.

This second major epidemic, and the third, occurred synchronously in all affected Districts (the above, plus Sibsagar), showing peaks of incidence in 1925

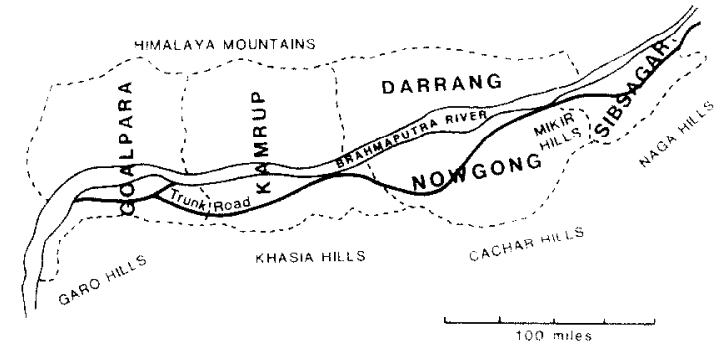

Fig. 1. Map of the Districts of Assam described in the text, modified from Rogers (1908). 
(i)
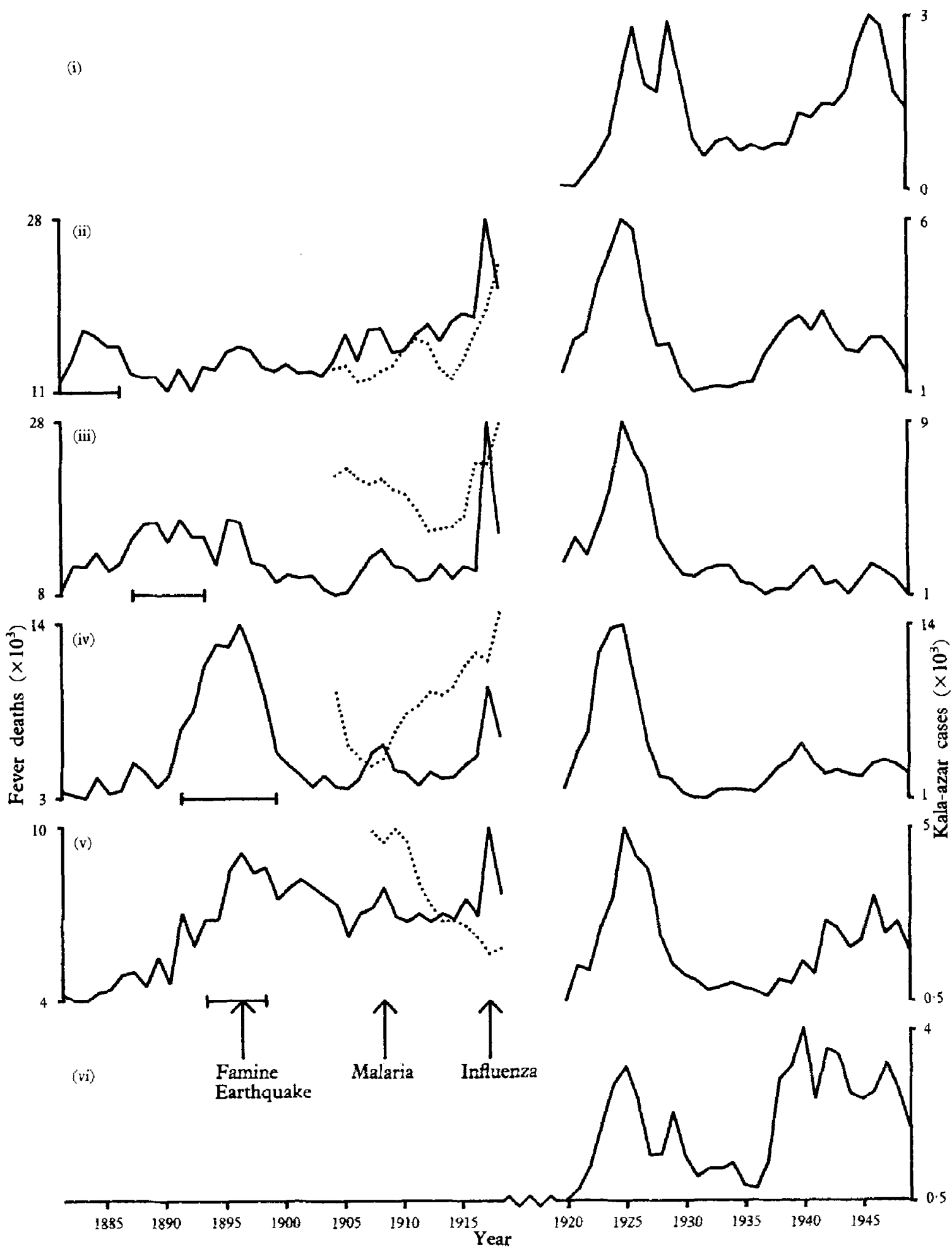

Fig. 2. Fever deaths (solid lines, left), kala-azar deaths (dotted lines) and kala-azar cases (solid lines, right) in the Districts of (i) Garo Hills, (ii) Goalpara, (iii) Kamrup, (iv) Nowgong, (v) Darrang, and (vi) Sibsagar between 1882 and 1949 . Kala-azar deaths are plotted with respect to the left hand scale running from 0-700. No data on deaths from fevers or kala-azar are available for the Garo Hills 1882-1920. 'The first epidemic did not reach Sibsagar. Horizontal bars mark the most important years of the first kala-azar epidemic according to RoGERS (1908). Arrows mark years when fever deaths were elevated by famine, earthquake, malaria and influenza. Data from Rogers (1908), McCombIe YOUNG (1924) and SEN GurTA (1951). 
and around 1945. Most cases recorded during the second epidemic occurred in the wake of the 19181919 influenza outbreak, although a rise in the kala-azar death rate predated influenza in at least 3 Districts. The second inter-epidemic period (interval between major peaks) was shorter and less variable than the first. The number of deaths due to kala-azar was undoubtedly highest during the first epidemic, but the number of cases may have been greater during the second (Fig. 5(i)).

\section{A Compartmental Model of Visceral Leishmaniasis}

To explain this pattern of epidemics, we begin by investigating the intrinsic processes. Extrinsic factors, such as other infectious diseases, can later be superimposed upon the basic pattern. Our analytical tool is a simple compartmental model which incorporates the essential biology of anthropoonotic (ASHFORD \& BETTINI, 1987) Indian $L$. donovani:

$$
\begin{aligned}
& \left.S_{t+1}=S_{t}+a N_{t}-(c N)_{t}^{x-1}\right) I_{t}\left(S_{t} / N\right)_{t}-b S_{t} \\
& I_{t+1}=I_{t}+\left(c N_{t}^{x-1}\right) I_{t}\left(S_{t} / N_{t}-(b+d) I_{t}\right. \\
& R_{t+1}=R_{t}+N_{t}-b R_{t}
\end{aligned}
$$

The total human population size $\left(N_{t}\right)$ is the sum of persons in 3 categories: susceptible $\left(S_{t}\right)$, infectious $\left(I_{t}\right)$ and resistant $\left(R_{t}\right)$. Infectious individuals $\left(I_{t}\right)$ all die from kala-azar. $R_{t}$ includes people totally resistant to infection, those who quickly and spontaneously recover after infection, and those who harbour parasites for many years without being clinically ill (NAPIER \& KRISHNAN, 1931; MOSKOVSKY \& SOUTHGATE, 1971; HEYNEMAN, 1971; REES \& KAGER, 1987). We assume that infectiousness is correlated with the severity of clinical signs, as it is for canine visceral leishmaniasis (RIoux et al., 1972), and that none of the various members of $R_{t}$ contribute, or have ever contributed, to the infectious pool $\left(I_{t}\right)$. In reality, resistants of the third kind can develop kala-azar when immunocompromised. Such persons may therefore contribute to local persistence of a parasite population, whilst having little influence on the dynamics under consideration here.

The natural death rate $b$ is much smaller than the disease-induced mortality $d$. These are two parameters for which reasonable, independent estimates can be obtained. In all simulations, we set $b$ to 0.037 following Christophers' statement (MCCOMBIE YouNG, 1924) that the expectation of life is 27 years at age $20 ; d=0.95$ is based on the observation that untreated kala-azar patients usually die within 1-2 years (MCCOMBIE YOUNG, 1924).

Susceptible and resistant individuals are born at rates $a$ and $e$ respectively. Available demographic data show the net growth rate of the human population to be very variable between Districts, and much influenced by death during kala-azar epidemics and immigration between them. In our simulations, $a+e-b$ varied between 0.018 and 0.025 . The latter, representing $2.5 \%$ growth per annum in the absence of deaths due to kala-azar, is approximately correct for Darrang during the decade (1912-1921) before the second epidemic (MCCOMBIE YoUNG, 1924). There is no substantial information with which we can specify the fraction of persons born resistant; that fraction was chosen to give the best fit to the data, e.g. $e /(e+a)=0.678$ in Fig. 5 .

Among models of insect-borne human diseases (MAY \& ANDERSON, 1979; ARON \& MAY, 1982; BAILEY, 1982), the transmission term of the system of equations (1)-(3) has two novel features. First, the total human population size is permitted to vary; over the 75 years of interest, kala-azar epidemics imposed significant reductions on a growing human population. Second, we introduce parameter $x$. Usually, $N_{t}$ is constant and the fly population is assumed to take a fixed number of bites in unit time, so $x=0$ and $c / N_{t}$ is the Vectorial Capacity (DYE, 1986). Here, however, we must acknowledge that the total number of infective bites is probably correlated with $N_{t}$, for Phlebotomus argentipes is a domestic sandfly which breeds in association with man and domestic animals (ASHFORD \& BETTINI, 1987; DYE, 1988). For simplicity, and in the absence of any data, we calculate the total number of infective bites arising from each person in a year as $c N_{t}^{x-1}$, with $x$ set at 1 (a constant number of sandflies per person). Constant $c$, like the fraction $e^{\prime}(e+a)$, is chosen to give the best fit to the data.

Sandfly seasonality and the incubation period in man suggest a time-step for the model of one year. The biting activity of flies reaches a maximum after the monsoon, between August and October (HATI, 1983). With flies biting at random over this period, a fraction $S_{\sqrt{ }} / N_{t}$ of these generate new cases. A new case will normally have an incubation period of 2-6 months (REES \& KAGER, 1987). Equating illness with infectiousness, we assume that cases will be infective to sandflies emerging the following year. However, the long-term dynamic behaviour of the model is very insensitive to variations of a few months in the latent period.

\section{Numerical Analysis}

Most theoretical investigations of periodicity in disease incidence deal with fluctuations around equilibrium (MAY \& ANDERSON, 1979; ANDERSON, 1982). Here, by contrast, we are concerned with the invasion of a new disease into a human populationwith the events which occur before any steady state is reached.

In Fig. 3, the model equations (1)-(3) are used to illustrate the consequences of introducing one infectious individual into a community, and the influence of the model's various parameters. The magnitude of $x$ is more important than Fig. 3(vi) reveals: set to its usual value of zero, the system is quickly damped after a first epidemic. Clearly, these numerical results have features in common with the data: in particular, there are, within 75 years, 3 epidemics with decreasing inter-epidemic period; it can take many more than 75 years to obtain stable fractions of susceptible, infective and resistant individuals.

To consider in detail the relationship between model and data, we can proceed with a District by District comparison, or treat the entire Province as a 

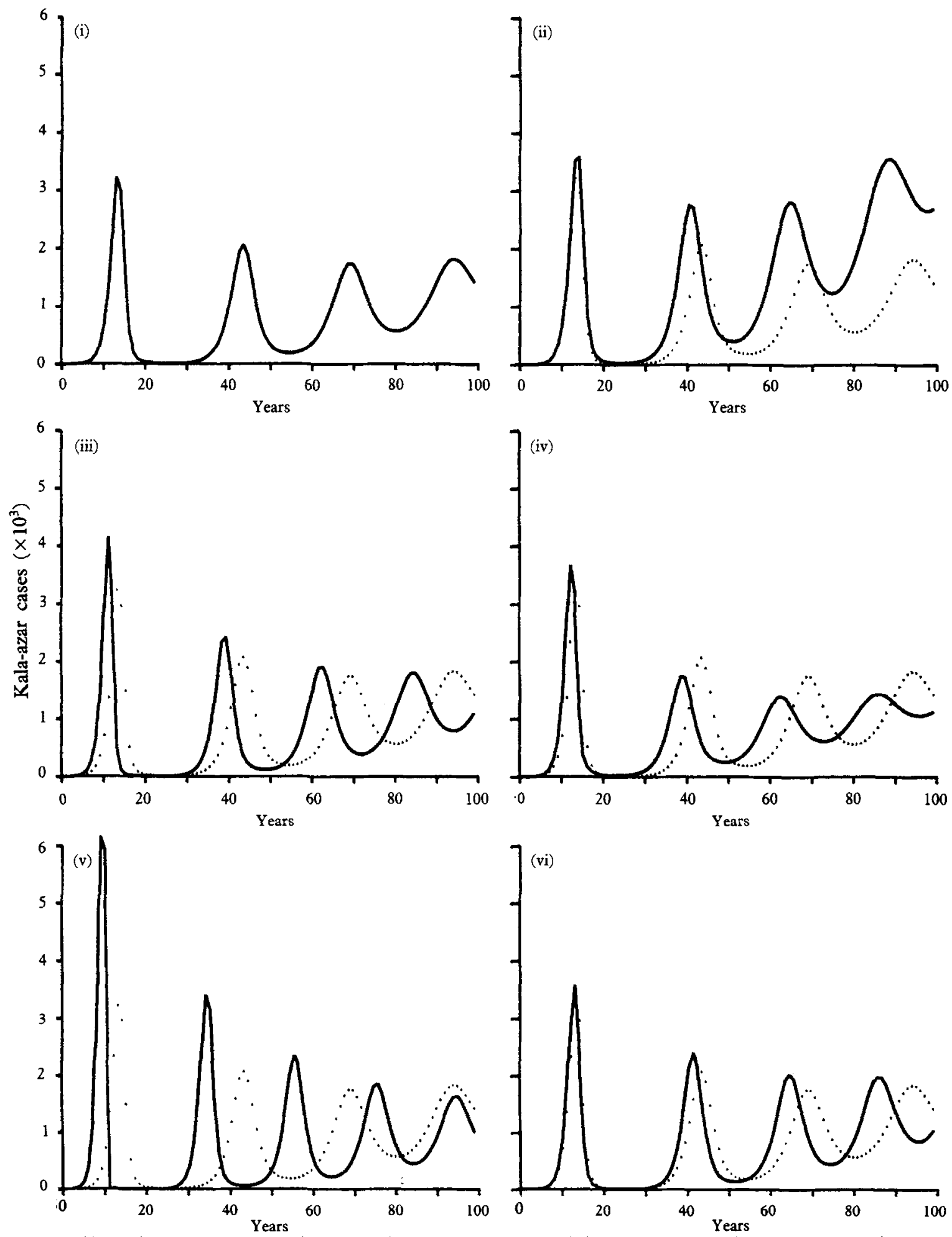

Fig. 3. Incidence of kala-azar for 100 years following the introduction of 1 infectious individual into a community, according to model (1)-(3). Parameter values in (i) are $a=0.0186, b=0.037, c=7, d=0.95, e=0.0434, x=1$ giving a basic reproductive rate of infection (ANDERsON, 1982) of $2 \cdot 13$. Other parameters yield the best fit to the data in Fig. $S(\mathrm{i}) . S_{0}=9 \times 10^{4}, R_{0}=2 \cdot 1 \times 10^{5}$ ensure that the initial structure of the population is compatible with the birth rates of resistant and susceptible persons; (ii) and (iii) show the effects of a $15 \%$ increase in $a$ and $c$, (iv) and (v) the effects of a $15 \%$ decrease in $d$ and $e$; in (vi) $x$ is increased by $15 \%$ while basic reproductive rate is held constant by decreasing $c$. The dotted line in (ii)-(vi) aids comparison with (i). 

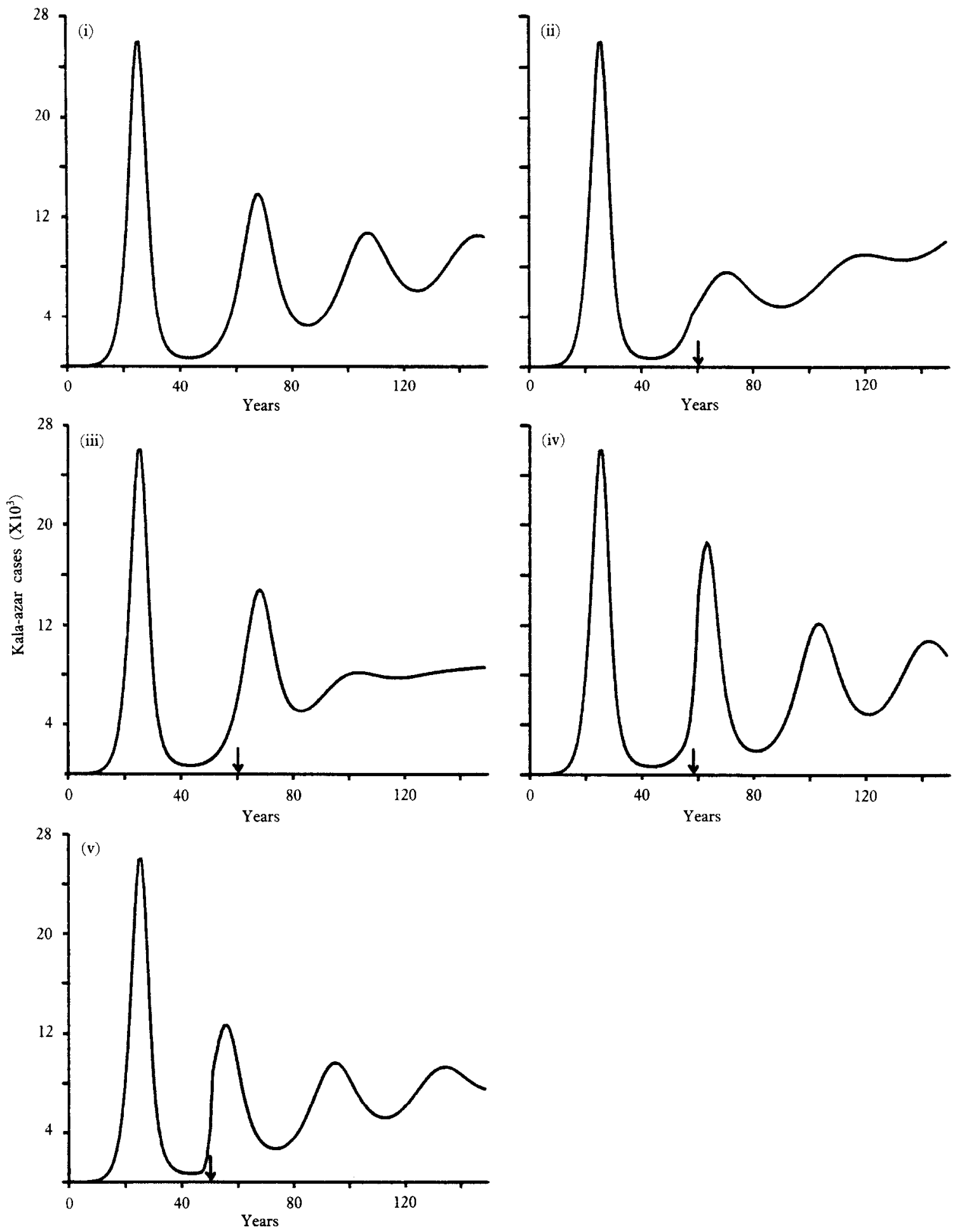

Fig. 4. (i) Attempts to reproduce with model (1)-(3) the change in the inter-epidemic period observed in Goalpara and Nowgong. Parameters $a=0.0275, b=0.037, c=3, d=0.95, e=0.0275, x=1$, initial values $S_{0}=1.5 \times 10^{5}, I_{0}=1, R_{0}=1.5 \times 10^{5}$ give a first inter-epidemic period of 40 years, equal to that observed in Goalpara, and a second inter-epidemic period of 39 years. (ii) Drug treatment beginning in year 60 (arrowed) reduces the average duration of infectiousness by transferring $15 \%$ of cases to the resistant class as soon as they become infected. (iii) Drug treatment beginning in year 60 gives $0.5 \%$ of cases a life expectancy of $1 / b$ rather than $1 / d$ years. (iv) $5 \%$ resistants become susceptible during years $58-62$ (first year arrowed), the possible consequence of an influenza epidemic. (v) $12 \%$ of resistant individuals become susceptible during years $50-54$, creating a first inter-epidemic period of 30 years, equal to that observed in Nowgong, but retaining a second inter-epidemic period of 39 years. 


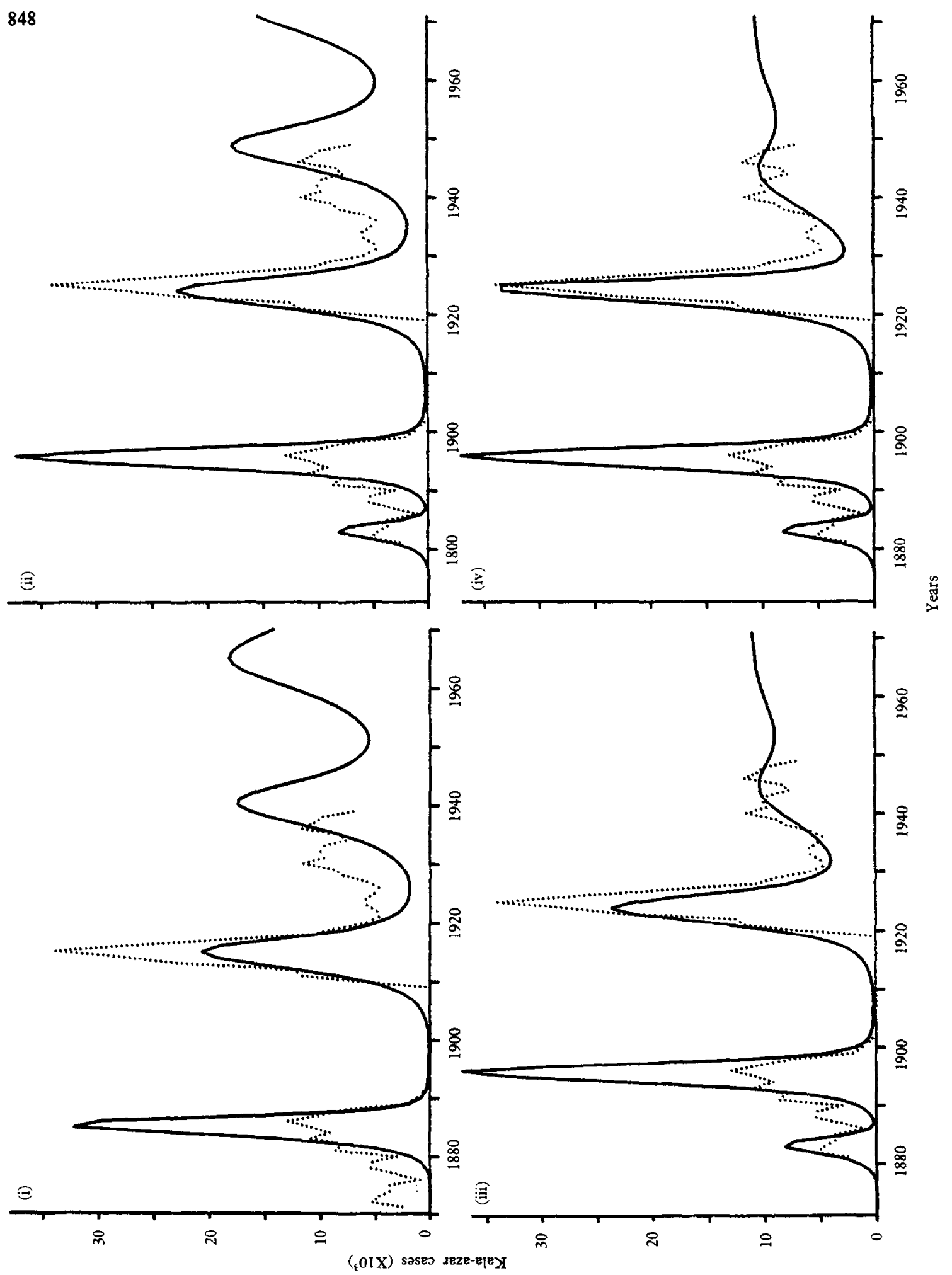

Fig. 5. Model (1)-(3) fitted to summed incidence cata for the Districts of Goalpara, Kamrup, Nowgong and Darrang. (i) Parameter values as in Fig. 2(i) selected to reproduce the timing of the first 2 epidemics. (ii) The spatial component of the first epidemic simulated by initially exposing $15-8 \%$ to infection, and the remaining $84 \cdot 2 \%$ in 1888 . (iii) Allowing for the sparial effect, but introducing chronic infectives ( $1 \%$ cases) from 1918 onwards. (iv) Allowing for the effects described in (ii) and iij) but with influenza exacerbating the second epidemic: $1 \%$ of resistant individuals become susceptible during 1921.1925. Parameter values in (ii)-(iv) are $a=0.019, b=0.037, c=7.5, d=0.95, e=0.04, x=1$. 
single entity. Taking the first course demands explanations for the Province-wide synchrony of the second and third epidemics, and for a ratio of inter-epidemic periods greater than 2:1 in the Districts of Goalpara and Garo Hills (Fig. 2(i,)(ii)). Fig. 4(i) shows how model (1)-(3) can reproduce Goalpara's first inter-epidemic period of 40 years. But we found no combination of parameter values capable of generating a second inter-epidemic period as short as 20 years.

At this point we acknowledge that our model accounts for none of the extrinsic factors which must have influenced the change in kala-azar incidence between 1885 and 1950 . Prominent among these was the introduction of antimonial drugs around 1920 . Intravenous administration of tartar emetic was a life-saving treatment; case mortality fell from $90 \%$ to $10 \%$ (MCCOMBIE YOUNG, 1924). Coverage was clearly wide: SEN GUPTA (1951) recorded 59997 cases in 7 Districts of Assam for the years 1920-1923, whilst MCCOMBIE YOUNG, (1924) estimated that 80035 persons in the Province were treated. With reference to model (1)-(3), the epidemiological significance of these drugs lies principally in their impact on the duration of infectiousness. For the majority of those treated the duration of infectiousness is likely to have been shorter. This would reduce the intensity of the second epidemic, increase the subsequent interepidemic period, and magnify the discrepancy between the model and the Goalpara data (Fig. 4(ii)). A smaller proportion of treated individuals, up to $25 \%$, eventually develop post kala-azar dermal leishmaniasis, a disfiguring skin disease which renders them chronically infectious to sandflies (REES \& KAGER, 1987). In theory, if just $0.5 \%$ of individuals remain durably infectious after treatment, the magnitude of the second epidemic would be marginally augmented and the third epidemic markedly damped (Fig. 4(iii)). The damping quickly converts an epidemic disease into an endemic one. In the process, the second inter-epidemic period is somewhat foreshortened, but the ratio of inter-epidemic periods can never be as large as $2: 1$. This sensitivity to the presence of a few extra infectives in the second inter-epidemic period reinforces the notion that they are of considerable epidemiological importance (NAPIER, 1943; ASHFORD \& BETTINI, 1987; REES \& KAGER, 1987).

The 1920-1930 epidemic of kala-azar was also preceded by widespread influenza (Fig. 2). MССoMBIE YOUNG (1924) believed that influenza was the principal cause of this epidemic, acting by increasing the fraction of the population which was susceptible to infection. A similar effect on resistant individuals has been ascribed to earthquakes, famine and malaria (ROGERS, 1908; NAPIER, 1943; SEN GUPTA, 1951). The process can certainly magnify an epidemic (Fig. 4(iv)), or generate an epidemic prematurely (Fig. $4(v)$ ), but it cannot shorten the second inter-epidemic period. Nor can the required ratio of inter-epidemic periods be obtained by simultaneously increasing susceptibility and introducing drugs. And we know of no other external force which, acting during the 1930s, might have propelled the third epidemic. The few data (Fig. 2(ii)-(iv)) describing the kala-azar death rate in Goalpara, Kamrup and Nowgong must also be weighed against MCCOMBIE YOUNG's (1924) view.
The death rate had begun to rise before the influenza epidemic of 1918-1919, in no particular relation to the malaria epidemic of 1908-1909. In summary, we cannot explain the timing of kala-azar epidemics in Assam by considering each District in isolation, and postulating a progenitive and synchronizing role for influenza.

A more successful approach treats, as a first approximation, the whole Province of Assam as a single unit with a thoroughly mixing human population (ignoring District boundaries). Migrant labour for the flourishing tea plantations would have contributed substantially to such mixing (MCCOMBIE YouNG, 1924).

The fitting process is subjective throughout. In Fig. 5 (i), for example, emphasis is given at first to the timing (see below) rather than the magnitude of the principal peaks of the first 2 epidemics. At each step, we choose combinations of parameters $c, e /(e+a)$ and $N_{0}$ and judge the performance of the model by eye. Our aim here is not necessarily to find the best possible combination of parameter values, but to see whether, using the Provincial rather than the District approach, any combination of reasonable parameter values can fairly represent the data.

Fig. 5(i) is the result of fitting model $(1)-(3)$ to summed incidence data for 4 Districts. Information describing the first epidemic is based on reports made by village headmen of deaths due to all fevers. Incidence figures were crudely obtained by subtracting baseline fever death rates mentioned by ROGERS (1908) from all fever deaths during the important kala-azar years (bars in Fig. 2), and assuming, with MCCOMBIE YOUNG (1924), that these constituted $90 \%$ of the cases arising in the previous year. These incidence figures accurately describe the timing of the first epidemic, but not its magnitude.

The actual duration of the frst epidemic was rather longer than its simulated counterpart, but unidirectional spread could explain the discrepancy. The spatial effect can be mimicked very simply by exposing the human population to infection in 2 stages (Fig. 5(ii)). Then, with a first inter-epidemic period of $30-40$ years, a small number of chronically infectious individuals, produced after 1919, can generate a second inter-epidemic period of 20 years followed by a smaller third epidemic (Fig. 5(iii)). Finally, the severity of the second epidemic could well have been exacerbated by widespread influenza (Fig. 5 (iv)).

\section{Conclusion}

The data presented by ROGERS (1908), MCCOMBIE YOUNG (1924) and SEN GUPTA (1951) show just 3 major epidemics in Assam between 1875 and 1950, with inter-epidemic periods of $30-45$ and 20 years. This interpretation of the early literature differs from the familiar idea of 10-20 year cycles, which may have arisen from a search for regularity.

The first point made by analysis with model (1)-(3) is that we should not be surprised that the second inter-epidemic period was shorter that the first: this is expected even without interference from drugs, natural disasters or other infectious diseases.

Unquestionably, the data used in this analysis are poor. But viewing them on 2 scales, the District and the Province, and over the full 75 years for which 
records are available, gives some of the leverage needed to separate the roles of intrinsic and extrinsic processes. The behaviour of model (1)-(3) is compatible with the view that the epidemic of the 1920 s struck a thoroughly mixing human population which was becoming increasingly susceptible to kala-azar. We cannot reconcile its behaviour with the view that the second epidemic was propagated and synchronized by widespread influenza in 1918-1919, though influenza may well have augmented the number of cases.

The third result of our analysis concerns the epidemiological importance of chronically infectious cases of visceral leishmaniasis. These may largely be patients suffering from post kala-azar dermal leishmaniasis (PKDL) - the unhappy sequel of life-saving drug treatment. Even a small fraction of cases developing PKDL could make disease more typically endemic than epidemic in a community. This point bears directly on discussion (e.g. INDIAN CoUNCIL FOR MEDICAL RESEARCH, 1983) about the epidemiological role of sub-clinically infectious individuals. Where such persons are numerous and fully infectious, we would not expect epidemic kala-azar.

Our results hinge on 3 important assumptions, 2 incorporated and one not incorporated in model (1)-(3): that a large fraction of the human population can resist developing fully infectious kala-azar, that sandfly population size depends upon human population size, and that the epidemic of the 1940s was not provoked by any extrinsic event. We reaffirm that the supporting evidence is minimal; it is qualitative with respect to the first 2 . In the case of the third, we have found no accounts to the contrary. However, the function of model (1)-(3), as with many simple models of infectious diseases, is not necessarily to be right, but to provide a framework for further systematic debate.

\section{Acknowledgements}

We thank C. R. Davies, R. Killick-Kendrick, R. P. Lane, R. C. Page and D. J. Rogers for helpful discussions. The work was supported, in part, by a grant from the UK Medical Research Council.

\section{References}

Anderson, R. M. (editor) (1982). The Population Dynamics of Infectious Diseases. London: Chapman \& Hall.

Aron, J. L. \& May, R. M. (1982). The population dynamics of malaria. In: The Population Dynamics of Infectious Diseases, Anderson, R. M. (editor). London: Chapman \& Hall, pp. 139-179.

Ashford, R. W. \& Bettini, S. (1987). Ecology and epidemiology: Old World. In: The Leishmaniases in Biology and Medicine, Peters, W. \& Killick-Kendrick, R. (editors). London: Academic Press, pp. 365-424.

Bailey, N. T. J. (1982). The Biomathematics of Malaria. London: Charles Griffin \& Co.

Dye, C. (1986). Vectorial Capacity: must we measure all its components? Parasitology Today, 2, 203-209.

Dye, C. (1988). Why measure the vectorial capacity of sandflies? In: Leishmaniasis: The First Centenary (18851985). New Strategies for Control, Hart, D. T. (editor). New York: Plenum, in press.

Hati, A. K. (1983). Current status of leishmaniasis-vector biology. In: Proceedings of the Indo-U.K. Workshop on Leishmaniasis. New Delhi: Indian Council for Medical Research, pp. 84-91.

Heyneman, D. (1971). Immunology of leishmaniasis. Bulletin of the World Health Organization, 44, 499-514.

Indian Council for Medical Research (1983). Proceedings of the Indo-UK Workshop on Leishmaniasis. New Delhi: Indian Council for Medical Research, pp. 173 \& 183.

Manson-Bahr, P. E. C. \& Apted, F. I. C. (1982). Manson's Tropical Diseases. London: Baillière Tindall.

May, R. M. \& Anderson, R. M. (1979). Population biology of infectious disease. Part II. Nature, 280, 455-46l.

McCombie Young, T. C. (1924). Kala-azar in Assam. London: H. K. Lewis \& Co.

Moskovsky, S. D. \& Southgate, B. A. (1971). Clinical aspects of leishmaniasis. Bulletin of the World Health Organization, 44, 491-497.

Napier, L. E. (1926). An epidemiological consideration of the transmission of kala-azar in India. Indian Medical Research Memoirs, 4, 219-267.

Napier, L. E. (1943). The Principles and Practice of Tropical Medicine. London: W. Thacker \& Co.

Napier, L. E. \& Krishnan, K. V. (1931). A theory of the aetiology and epidemiology of kala-azar in India. Indian Medical Gazette, 66, 603-609.

Peters, W. \& Prasad, L. S. N. (1983). Kala-azar in India-its importance as an issue in public health. In: Proceedings of the Indo-U.K. Workshop on Leishmaniasis. New Delhi: Indian Council for Medical Research, pp. 5-9.

Rees, P. H. \& Kager, P. A. (1987). Visceral leishmaniasis and post kala-azar dermal leishmaniasis. In: The Leishmaniases in Biology and Medicine, Peters, W. \& KillickKendrick, R. (editors). London: Academic Press, pp. 583-615.

Rioux, J.-A., Lanotte, G., Croset, H. \& Dedet, J.-P. (1972). Ecologie des leishmanioses dans le sud de la France. 5. Pouvoir infestant comparé des diverses formes de leishmaniose canine vis-à-vis de Phlebotomus ariasi Tonnoir, 1921. Annales de Parasitologie Humaine et Comparée, 47, 413-419.

Rogers, L. (1908). Fevers in the Tropics. London: Henry Frowde.

Sanyal, R. K. (1985). Leishmaniasis in the Indian subcontinent. In: Leishmaniasis, Chang, K.-P. \& Bray, R. S. (editors). Amsterdam: Elsevier, pp. 443-468.

Sanyal, R. K., Banerjee, D. P., Ghosh, T. K., Ghose, J. N., Misra, B. S., Roy, Y. P. \& Rao, C. K. (1979). Longicudinal review of kala-azar in Bihar. Foumal of Communicable Diseases, 11, 149-169.

Sen Gupta, P. C. (1951). A report on kala-azar in Assam. Indian Medical Gazette, 86, 266-271 \& 312-317.

Shortt, H. E. (1945). Recent research on kala-azar in India. Transactions of the Royal Society of Tropical Medicine and Hygiene, 34, 13-31.

Received 25 February 1988; accepted for publication 25 April 1988 